\title{
Long-term Complications after COVID-19 Infections Optimal Management Remains Unclear - International Cross-Sectional Collaboration Is Necessary
}

\section{Hanne Tønnesen}

doi.org/10.29102/clinhp.21001

\section{About the AUTHOR}

\section{Hanne Tønnesen}

Editor-in-Chief

Director, Clinical Health

Promotion Centre, Bis-

pebjerg \& Frederiksberg

Hospital, University of

Copenhagen, Copenhagen,

Denmark.

Professor at Lund Universi-

ty, Skåne University Hospi-

tal, Malmö, Sweden

Contact:

Hanne Tønnesen

Hanne.Tonnesen@regionh.dk

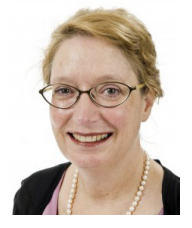

Clin Health Promot 2021; 11:3-4

\section{The new problem among survivors after Covid-19 infections}

The infectious coronavirus disease 2019 (Covid-19) pandemic is caused by the severe acute respiratory syndrome coronavirus (SARS-CoV-2). Until now, it has led to more than 2 million registered deaths, worldwide. Many more persons survived the infection, but several are suffering from a large variety of long-term complications, especially respiratory problems as well as fatigue, exercise intolerance, and poor concentration (1). Recently, a wide range of symptoms and severity have been described too, including local pain from one or more organ systems, nausea, skin rashes, anxiety, depressions, and delirium (2).

Treatment at Intensive Care Units is associated with more and longer dysfunctions at 6-month follow-up. The frequency of the long-term complications has been described to be up to $70 \%$ among the hospitalized patients, who have more severe symptoms and are better observed compared to those treated outside the hospitals, with milder symptoms and without a verified diagnosis of Covid-19 (3).

The long-term complications are not restricted to adults, as it seems that children would experience similar long-term effects after the infections (4).

Though the possible correlation between the severity of the infection and the symptoms of long-term complications is not fully described today, there is no doubt that long-term complications after Covid-19 infections exist and impact the recovery and the quality of life. They have become a new challenge in the treatment of Covid-19.
Sparse evidence for diagnosing, treatment, outcome, and prevention of long-term complications Until further, the evidence of effective programs for diagnosing, treating, follow-up or preventing the long-term complications are sparse. The overall treatment consists of symptom-based interventions, which is in accordance with the usual routines for management of diseases with no or only weak evidence-based treatment. The guidelines are frequently updated, but it is a relatively new condition, and more evidence is required, still.

Today, the general recommendations aim to distinguish between an ongoing Covid-19 infection, long-term complications and unrelated diagnoses. They include a detailed history, a chest $\mathrm{x}$-ray, and blood sampling to be analyzed for infectious response and kidney, liver, thyroid functions. Other examinations are tailored to the symptoms of the individual patients (5). Furthermore, the measurement of the outcome requires close follow-up as described in the recommendation, which however, does not yet include a preventive focus.

Continuous communication, shared decision making, and close collaboration between patients and staff are very important elements in the management of diseases without clarified diagnosing, treatment, and outcomes. Be aware of the broad need for better health literacy (6), both because the sparse knowledge may be difficult to deal with for both staff, patients, and relatives, and because the Covid-19 pandemic seems to have a social gradient that may be reflected in the long-term complications as well. 


\section{Unhealthy lifestyles}

The impact has not yet been fully investigated, but an increasing amount of knowledge indicates that unhealthy lifestyle such as smoking and underlying diseases are associated with progression and severity of Covid-19 $(7 ; 8)$. An aggravation of long-term complications by smoking could be expected for Covid-19 similar to other pulmonary infections (9); however, the mechanisms are not clearly described. Another association has been identified between obesity and severity of the outcomes (10). Also, vitamin D insufficiency may play a role (11).

Though it is common sense to improve unhealthy lifestyles and compensate for avitaminoses, it is necessary to evaluate if there is an extra clinical effect of acute lifestyle intervention on the outcome and prevention of long-term complications after the Covid-19 disease similar to the rather fast effect of lifestyle intervention in lowering development of complications after e.g. surgery, pregnancy, and noncommunicable diseases.

\section{From fragmented knowledge on to establish- ment of new evidence}

The first step in gathering new evidence is to systematically collect the relevant data and patient reported information in order to develop qualified new hypotheses. The following step, is to test and evaluate the hypotheses in high-quality design studies.

The first step involves establishment of new cohorts of patients with Covid-19 infections. This seems absolutely do-able based on systematic data collection from a broad range of high, middle, low, and very low-income countries.

A well-known challenge would be to collect the data in regions without the ability to test, treat, follow-up, and register the individual patients. Another challenge originates from countries not wanting to share their real-life data with the international research society. The World Health Organization would be a natural and very experienced leading partner to facilitate the collection and sharing of data among states.

Several high-income countries already have large population cohorts to build on. To develop preventive hypotheses, the cohorts should include both the patients developing the long-term complications as well as those not developing the complications.

Due to the relatively short duration of the pandemic, most of the Covid-19 studies on rehabilitation are based on observations, some of which are associated with pathophysiological changes, but correlations should not be confused with causality.

\section{Collaboration is strongly required}

During the pandemic, we have witnessed the effectiveness of new and close collaborations. An important collaboration has been established between the private and public organisations regarding development and test of new vaccines. This collaboration is driven by the very high economic benefits for the industry as well as the entire society, the healthcare system, and the individuals - in addition to the general focus on health and survival.

New strong collaborations are taking place among groups with multidisciplinary participants across the world to develop, follow-up and disseminate new knowledge on Covid-19 facilitated by extra focused public and private funding and open fast-track publication amongst others.

It is necessary to build on and extend these collaborations to include the development of international evidence-based guidelines on diagnosing, treatment, follow-up and prevention of long-term complications for the benefit of the individuals, the healthcare system and the society at large (with both public and private organizations).

Overall, the new and continuously updated evidence-based clinical guidelines shall build on results from studies with explicit hypotheses that has been evaluated in high quality designs like randomized clinical trials with nested pathophysiological and qualitative evaluations. The implementation of these guidelines requires follow-up for effect in real life in clinical registries. The best way to do this is to collaborate!

\section{References}

(1) Fraser E. Long Term Respiratory Complications of COVID-19. BMJ 2020;370:m3001. doi:10.1136/bmj.m3001.

(2) Shah W, Hillman T, Playford ED, et al. Managing the Long Term Effects of COVID-19: Summary of NICE, SIGN, and RCGP Rapid Guideline. BMJ 2021;372:n136. doi:10.1136/bmi.n136.

(3) Himmels JPW, Qureshi SA, Brurberg KG, et al. COVID-19: Long-Term Effects of COVID-19. Norwegian Institute of Public Health 2021. ISBN (digital): 978-82-8406-175-7.

(4) Ludvigsson JF. Case Report and Systematic Review Suggest That Children May Experience Similar Long-Term Effects to Adults after Clinical COVID-19. Acta Paediatr. 2021;110:914-21. doi:10.1111/apa.15673.

(5) Office for National Statistics. The prevalence of long COVID symptoms and COVID-19 complications. 2020. www.ons.gov.uk/news/statementsandletters/theprevalenceoflongcovidsymptomsandcovid19complications.

(6) Pelikan JM, Ganahl K, Roethlin F. Health Literacy as a Determinant, Mediator and/ or Moderator of Health: Empirical Models Using the European Health Literacy Survey Dataset. Glob Health Promot. 2018;25:57-66. doi:10.1177/1757975918788300.

(7) Patanavanich R, Glantz SA. Smoking Is Associated With COVID-19 Progression: A Meta-analysis. Nicotine Tob Res. 2020;22:1653-6. doi:10.1093/ntr/ntaa082.

(8) Guo FR. Smoking Links to the Severity of COVID-19: An Update Of a Meta-Analysis. J. Med. Virol. 2020; 92: 2304-5. doi:10.1002/imv.25967.

(9) Kashyap VK, Dhasmana A, Massey A, et al. Smoking and COVID-19: Adding Fuel to the Flame. Int J Mol Sci. 2020;21:6581. doi:10.3390/ijms21186581.

(10) Senthilingam M. COVID-19 Has Made the Obesity Epidemic Worse, but Failed to Ignite Enough Action. BMJ 2021;372:n411. doi:10.1136/bmj.n411. (11) Munshi R, Hussein MH, Toraih EA, et al. Vitamin D Insufficiency as a Potential Culprit in Critical COVID-19 Patients. J Med Virol 2021;93:733-40. doi:10.1002/jmv.26360. 